\title{
Quantification of subjective scaling of friction using a fingertip biomechanical model
}

\author{
Mohammad Abdolvahab \\ Department of Computer Science, University of Sciences and Technology of Lille, \\ Cité Scientifique, Villeneuve d'Ascq, France
}

\begin{abstract}
Subjective scaling of friction is important in many applications in haptic technology. A nonhomogeneous biomechanical finite element model of fingertip is proposed in order to predict neural response of sensitive mechanoreceptors to frictional stimuli (Slowly Adapting SAII receptors under the glabrous skin). In a guided psychophysical experiment, ten human subjects were asked to scale several standard surfaces based on the perception of their frictional properties. Contact forces deployed during the exploratory time of one of the participants were captured in order to estimate required parameters for the model of contact in the simulation procedure. Consequently, the strain energy density at the location of a selective mechanoreceptor in the finite element model as a measure of discharge rate of the neural unit was compared to the subject's perceptual evaluation of the relevant stimuli. It was observed that the subject's scores correlate with the discharge rate of the given receptor.
\end{abstract}

Keywords: Tactile, finite-element model, skin mechanoreceptors, friction perception

\section{Introduction}

With the advent of haptic technologies and applications, mechanistic study of sense of touch has been widely considered by researchers. Different applications in medical simulation, development of tactile devices and virtual environments demand elaborate quantification of perceptual mechanism dealing with the tactile sense. For example, during a laparoscopic surgery many judgements rely on how the surgeon feels a tissue, or when it is desired to deliver tactual information to a user exploring a tactile display using his/her fingertip. Subjective scaling of frictional forces is essential in many applications like estimation of required grasping force or rendering tactile images by means of variable friction tactile displays [1]. The relation between neural activities of relevant

\footnotetext{
*Corresponding author: Mohammad Abdolvahab, Department of Computer Science, University of Sciences and Technology of Lille, Cité Scientifique, 59655, Villeneuve d'Ascq, France. E-mail: mabdolva@gmail.com.
}

mechanoreceptors responding to frictional stimulus and subjective perception is sought in the present study using a biomechanical model of fingertip.

Classically, tactile perception is presumed as the result of trains of neural spikes caused by local deformation of mechanoreceptors under the fingerpad skin when the human being moves his finger on a surface during an exploration task. Four different types of mechanoreceptors have been identified that are sensitive to different mechanical stimuli $[9,10]$. It is widely known that SAI mechanoreceptors are responsible for perception of surface geometrical features like roughness or texture while SAII fibers mostly respond to tangential skin stretch. Beside these, Rapidly Adapting mechanoreceptors respond to vibrational stimuli within their sensitivity range [9]. All of these peripheral neural units transmit coded information about sense of touch to the central nervous system, where after complex processing procedures, a perceptual image of the mechanical features of the objects in contact is portrayed. 
Since mechanical contact information is transmitted through the fingerpad's skin before reaching mechanoreceptors, study of biomechanical response of skin and subcutaneous tissues is fundamentally important. Mechanical models predicting response of various mechanoreceptors have already been proved to be capable of estimating neurophysiologic empirical results [14] (Dandekar et al., 2003). In this paper, a 2-D biomechanical model of the human fingerpad is proposed in order to compare quantified response of SAII receptors identified with Ruffini endings, which play an important role in friction perception, to subjective scaling of relevant frictional stimuli. Such a strategy, i.e. comparison between the response of the mechanical model and psychophysical experimental results, is a useful methodology in tactile sense research in the absence of neurophysiological data.

\subsection{Mechanical modelling of fingerpad}

In spite of a vast amount of effort invested in the modelling of the fingerpad through various approaches, from focusing on precise geometrical configuration to identification of material properties of fingerpad tissues, there is little work investigating perceptual tactile phenomena employing the proposed models. This is probably due to obscure mechanoreceptive procedures or limitations on classical interpretations of peripheral and central nervous system operation or theories of psychophysics.

Irrespective of complexities in the psychophysics of the sense of touch, however, mechanical correlates of empirical neurophysiological data of individual mechanoreceptor firings have already been investigated. Dandekar et al. (2003) developed 3-D finite element model of human and monkey fingertips, following their previous 2-D models [14], with realistic geometrical features and showed that a multilayered model was able to predict response of slowly adapting type I mechanoreceptors (SAI) to indentations by complex object shapes. Wu et al. [21] investigated response of a 2-D model consisting of all major anatomical substructures (skin, subcutaneous tissue, bone, and nail) to vibrational stimuli. They concluded that vibration induced dynamic strain distribution at different depths in the fingerpad subcutaneous structure was dependent on the frequency range, and that these results for local mechanoreceptors (RAI or RAII) were consistent with the experimental observations for vibrotactile perception in the literature. Maeno et al. [12] proposed a 2-D inhomogeneous finite element model of the human finger but no correspondence with neurophysiological empirical results was reported.

\subsection{Friction perception and SAII receptors}

Perception of frictional cues has been of interest in many applications. Johansson and Westling $[6,7]$ analyzed the roles of glabrous skin receptors in automatic control of precision grip for lifting rougher or more slippery objects with the human hand. In particular, many researchers believe that SAII mechanoreceptors are the most likely source to provide the neural system with information about skin stretch [3, 10, 15, 19]. These large endings (dimensions of up to 200 by $1000 \mu \mathrm{m}$ ) are located in the dermis and respond to small displacements of the skin either directly over the receptor (threshold of about $15 \mu \mathrm{m}$ ) or as a result of stretch of the skin from a distance [20]. Harrington and Merzenich [5] investigated that an increase in the skin displacement intensifies the discharge rate of a typical receptor. Smith et al. (1996) proposed another strategy to study the subjective evaluation of friction. They used different surfaces to relate subjective sense of friction to measured contact forces and concluded that subjects were able to scale different tangential forces exerted on the fingerpad skin.

In this study, a similar combination of psychophysical experiment and measurement system is set up. The biomechanical finite element model of finger-surface contact is then employed to predict neural response of a typical SAII mechanoreceptor with captured contact information as input parameters. Numerical approximations are then compared to psychophysical experimental results.

In section 2, a psychophysical experiment is proposed that provides quantification of subjective judgements on two different tactile aspects directly concerned with friction perception. Section 3 is dedicated to the experimental setup that feeds the proposed mechanical model with contact parameters ruling over subjective judgements. In section 4 , different issues concerning the modelling of fingerpad structure are discussed. In the last section, the model response to various frictional stimuli is examined to investigate correlation of mechanistic response of relevant mechanoreceptors with subjective scales of friction. 


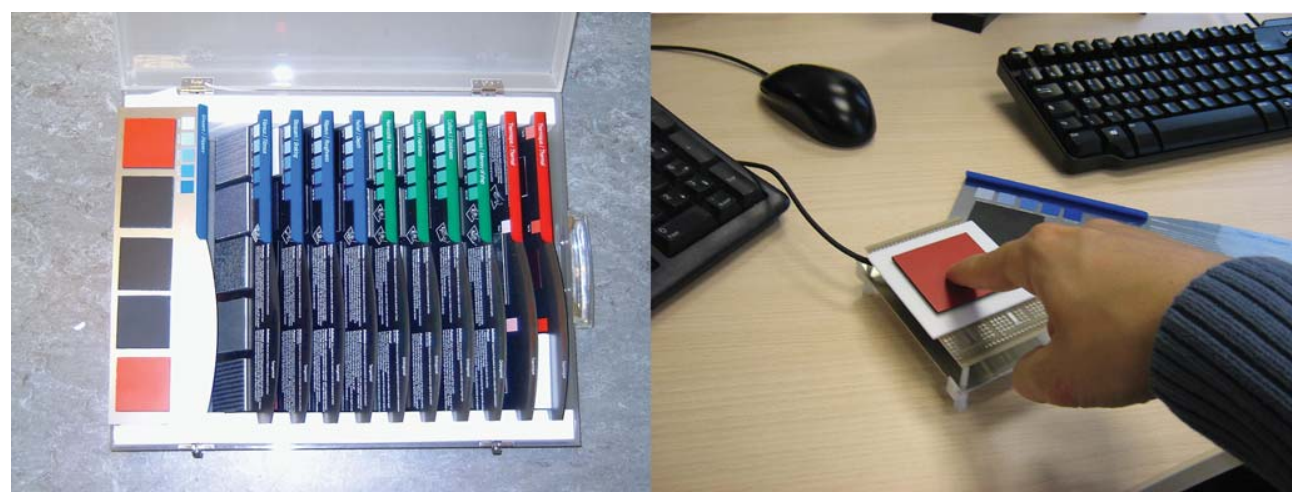

Fig. 1. Left: Sensotact, Right: Measurement setup for capturing contact forces during subjects' exploration.
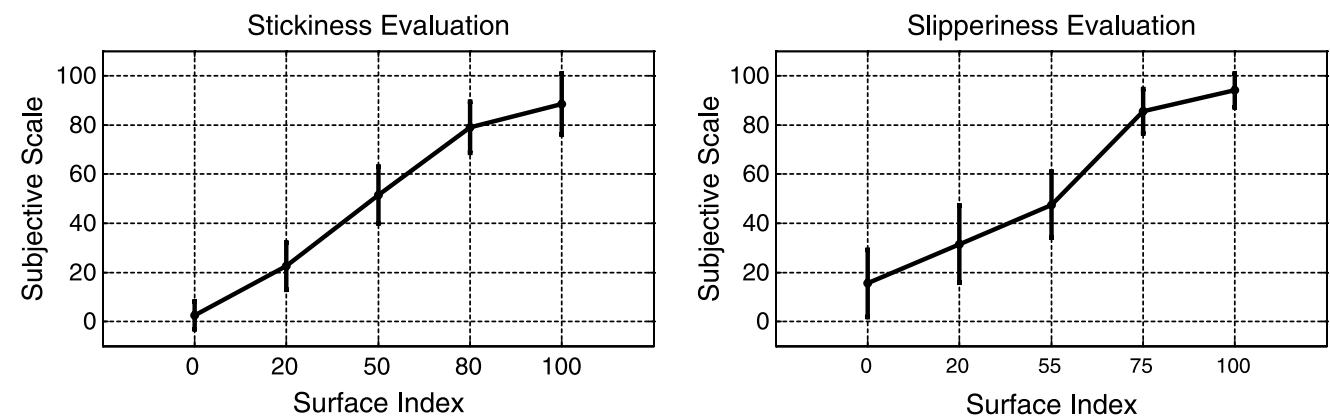

Fig. 2. Mean subjective scales for different samples; Error bars represent 95\% confidence interval.

\section{Psychophysical experiments}

In order to assess subjective scaling of friction of different stimuli, Sensotact ${ }^{\circledR}$ [2] was employed. Sensotact ${ }^{\circledR}$ is a suitcase composed of 10 descriptors and 50 references (Fig. 1, left). This sensorially preevaluated experimental tool is divided into several categories, each of them permitting assessment of different dimensions of tactile sense (based on static, orthogonal and tangential movement of fingertip).

In the present study, five samples of each of "sticky" and "slippery" descriptors were used to find the relation of mechanical contact between the subject's fingerpad and surfaces in a dynamic exploration to his perceptual evaluation of friction. The samples are primarily graded by the producer according to relevant standardized sensorial analysis. However, a pre-evaluation test with the same guidelines stated on the descriptors, was carried out to assure consistency of the grading system. Ten subjects were asked to use their index finger to stroke each sample and score it as a number between 0 and 100 . According to the instructions, the judgement was based on actively touching the surface in a distal-to-proximal direction for slipperiness evaluation and in a proximal-to-distal course for the assessment of stickiness (as shown in Fig. 1), while maintaining the contact angle between finger axis and surface at $45^{\circ}$. The subjects were free to use a speed of their choice to stroke each surface.

Figure 2 shows the results of the experiment for both of the stickiness and slipperiness judgements of all subjects. It was expected that subjects evaluate the stickiest surface (\#100) by the highest score similar to the most slippery one in the slipperiness category (\#100). The sticky or slippery properties of related samples were scaled by numbers very close to the company index numbers (marked on horizontal axis).

\section{Experimental measurement of deployed exploratory forces}

Fingertip contact forces during the tactile exploration of surfaces described in the previous section were experimentally measured using a six-axis transducer (ATI Nano43). These captured data were 
employed to provide the computational finite element model of contact with realistic mechanical parameters including tangential forces and friction coefficient of fingertip-surface contact.

The measurement was done by means of a magnetic surface glued to a fixed plate on the sensor. Sample surfaces were easily replaceable by affixing them to a magnetic base. During the exploration tasks, fingertip forces could not overcome this magnetic coupling. One of the subjects, who had taken part in the psychophysical experiment, was asked to repeat his trial, with the same guidelines, this time on the surfaces mounted on the transducer. Only one exploratory movement was permitted for this part. Sampling frequency was set on $200 \mathrm{~Hz}$ allowing all forces and torques $\left(\mathrm{F}_{\mathrm{x}}, \mathrm{F}_{\mathrm{y}}, \mathrm{F}_{\mathrm{z}}\right.$, $\mathrm{T}_{\mathrm{x}}, \mathrm{T}_{\mathrm{y}}, \mathrm{T}_{\mathrm{z}}$ ) to be captured as well. Data collection and storage was started just before subject touched the surface. After implementing the procedure for all surfaces, some post-processing work was carried out on raw data to remove useless captured samples before and after the contact. Contact forces and frictional coefficients, $\mu$, as the ratio of the tangential force to the normal force $\left(\sqrt{ }\left(\mathrm{F}_{\mathrm{x}}^{2}+\mathrm{F}_{\mathrm{y}}^{2}\right) / \mathrm{F}_{\mathrm{z}}\right)$ are shown in Figs. 3 and 4 for different samples.

Deployment of forces during the contact of fingerpad with different samples is presented by distinguished colors in the figures. In the stickiness assessment test, as can be observed in Fig. 3, variation of coefficient of friction is divided into two phases
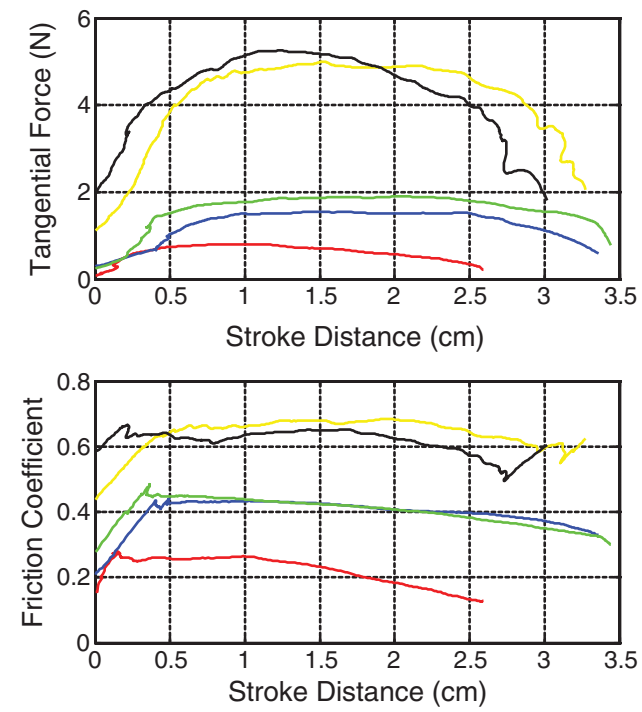

because of the blocking effect of the sample surface and the direction of fingertip movement: A linearly increasing phase before reaching to the critical friction coefficient when it switches to a relatively steady dynamic friction coefficient that can be approximated to be equal to the critical one. This phenomenon is not seen in the second experiment because of the lack of the sticky effect and the facility of fingertip movement in the inverse direction. However in both experiments, maximum tangential force values comply with index numbers of surfaces (i.e. the less sticky the surface the less tangential force made for the sticky category and the less slippery the surface the more tangential force resulted) while normal forces did not necessarily obey the rule because of the subjective dependence. Nevertheless, the friction coefficient computed for sample \#55 was lower than \#75 in the slippery category for subjects in most of the course, unexpectedly (probably because of moisturizing of the skin), as the former is evaluated as less slippery.

The choice of parameters required to feed the model is crucial to bridge the real world observation and psychophysical phenomenon of interest. The proposed finite element model in the next section is constructed using Ansys contact technology that is based on Coulomb friction model. Major required parameters for the analysis consist of coefficient of friction, $\mu$, and maximum contact friction, $\tau_{\max }$, with units of stress. By definition, this maximum contact friction stress can

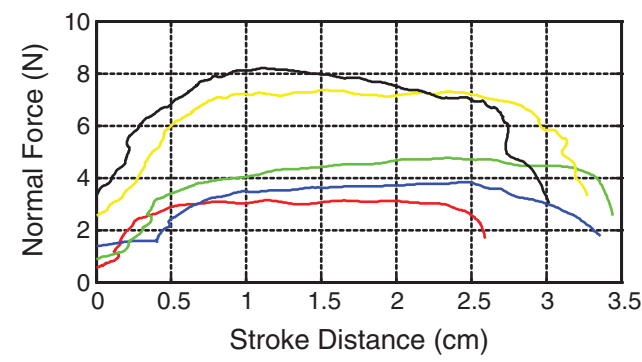

$\begin{aligned} & \text { Deployment of forces in exploratory } \\ & \text { evaluation of braking effect }\end{aligned}$
$\begin{aligned} & { }^{\star *} \text { Curve colors correspond to different } \\ & \text { sample surfaces with index numbers }\end{aligned}$
$-\# 20$
$-\# 80$
$-\# 100$

Fig. 3. Deployment of fingertip forces in contact with standard surfaces (stickiness assessment experiment) - different colors correspond to different sample surfaces with indicated index numbers (same as psychophysical experiment). 

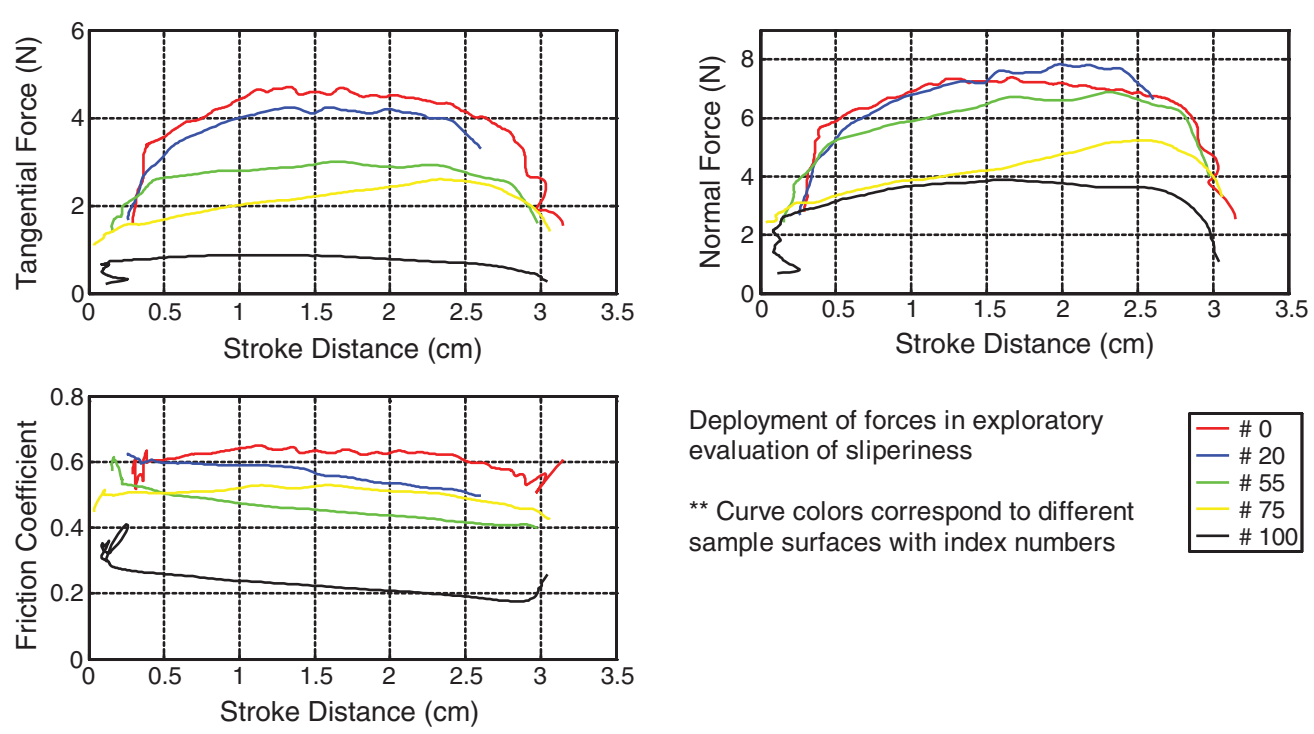

Deployment of forces in exploratory evaluation of sliperiness

** Curve colors correspond to different sample surfaces with index numbers

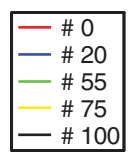

Fig. 4. Deployment of fingertip forces in contact with standard surfaces (slipperiness assessment experiment) - different colors correspond to different sample surfaces with indicated index numbers (same as psychophysical experiment).

be introduced so that, regardless of normal contact pressure, sliding will occur if the friction stress reaches this value. The value of $\tau_{\max }$ should be determined empirically.

With respect to the present experiment, this value was approximated by dividing the maximum tangential force during the exploration course by the effective contact area of subject's fingerpad $\left(\sim 1 \mathrm{~cm}^{2}\right)$. Maximum coefficients of friction for the sticky assessment experiment that were approximated equal to the dynamic friction coefficients were chosen to feed the model. In the second experiment, as the subject's fingertip immediately reached active movement on the sample, the dynamic friction coefficient was approximated by its mean over a steady range during the course (refer to Fig. 4).
Tables 1 and 2 show the data to serve the model. The speed of fingertip movement, another factor mentioned in the tables, is computed by averaging instantaneous velocity, Fig. 5, over a range of samples where less fluctuation was observed.

\section{Biomechanical model response}

The finite element modelling of the fingertip consists of two major tasks; geometrical modelling and determination of biomechanical properties of subcutaneous tissues.

Unlike the precise geometry of a finite element model, which is not widely studied in the literature, different attempts have been carried out to

Table 1

Sticky evaluation experiment

\begin{tabular}{llllll}
\hline Index no. of surface & 0 & 20 & 50 & 80 & 100 \\
\hline$\mu$ & 0.28 & 0.44 & 0.48 & 0.64 & 0.67 \\
$\tau_{\max } \times 10^{-4}(\mathrm{MPa})$ & 0.8033 & 1.5478 & 1.9038 & 4.9998 & 5.255 \\
$\mathrm{~V}(\mathrm{~cm} / \mathrm{s})$ & 2 & 2.2 & 2.1 & 1.75 & 1.5 \\
\hline
\end{tabular}

Table 2

Slipperiness evaluation experiment

\begin{tabular}{llllll}
\hline Index no. of surface & 0 & 20 & 55 & 75 & 100 \\
\hline$\mu$ & 0.62 & 0.55 & 0.44 & 0.52 & 0.24 \\
$\tau_{\max } \times 10^{-4}(\mathrm{MPa})$ & 4.707 & 4.2404 & 3.009 & 2.6 & 0.877 \\
$\mathrm{~V}(\mathrm{~cm} / \mathrm{s})$ & 1.3 & 3.3 & 3.9 & 3.8 & 3.9 \\
\hline
\end{tabular}


Table 3

Material properties of fingerpad's layers

\begin{tabular}{lcccr}
\hline & Epidermis & Dermis & Subcutaneous fat & Bone \\
\hline Young modulus $(\mathrm{MPa})$ & 0.136 & 0.08 & 0.034 & 200 \\
Poisson ratio & 0.48 & 0.48 & 0.48 & 0.3 \\
\hline
\end{tabular}

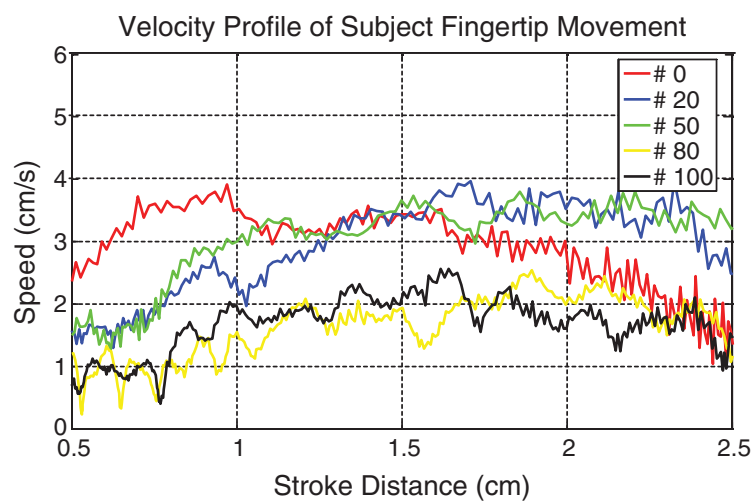

Fig. 5. Velocity variation during the subject's fingertip movement over the samples for the stickiness evaluation.

identify physical parameters of fingerpad structure. Most of these approaches are founded on in-vivo load displacement experiments to identify biomechanical parameters of skin (Dandekar et al. 2003) [11, 16, 18].

As a general conclusion from different models reported in the literature, the fingerpad may be supposed to be composed of three major layers; Epidermis, Dermis and fat subcutaneous tissue in addition to the bone. Dandekar et al. (2003) concluded that their homogenous model failed to predict the deformation data under line load irrespective of the model dimension. They investigated magnitude order of elasticity modulus of layers of fingerpad by verification of force-displacement measurements with empirical observed data.

Hence, the proposed 2D finite element model in this paper (Fig. 6, right) consists of four sublayers with schematic geometrical features. Approximate dimensions were estimated from a CT image of fingertip (Fig. 6, Left). In order to estimate dimensions of the model, it was assumed that fingertip is closely a cylinder with diameter $10 \mathrm{~mm}$ as a scale and other features were approximated based on the CT image. In particular, the thicknesses of epidermal and dermal layers were approximately 0.75 and $1.25 \mathrm{~mm}$, respectively. Numerical values of physical parameters used are those of the model introduced by Maeno et al. [12] given in Table 3 that comply with reported values by Dandekar et al. (2003) in the order of magnitude.

\subsection{Dynamic touch simulation and prediction of mechanoreceptor's responses}

Simulation of the fingerpad-surface contact was carried out by ANSYS contact technology. Visco-solid triangular elements were used because of their flexibility in the contact analysis. The solution of the problem was carried out on the static mode, however by dividing the solution into several substeps, details of computations can be displayed dynamically. Among various numerical algorithms, the Augmented

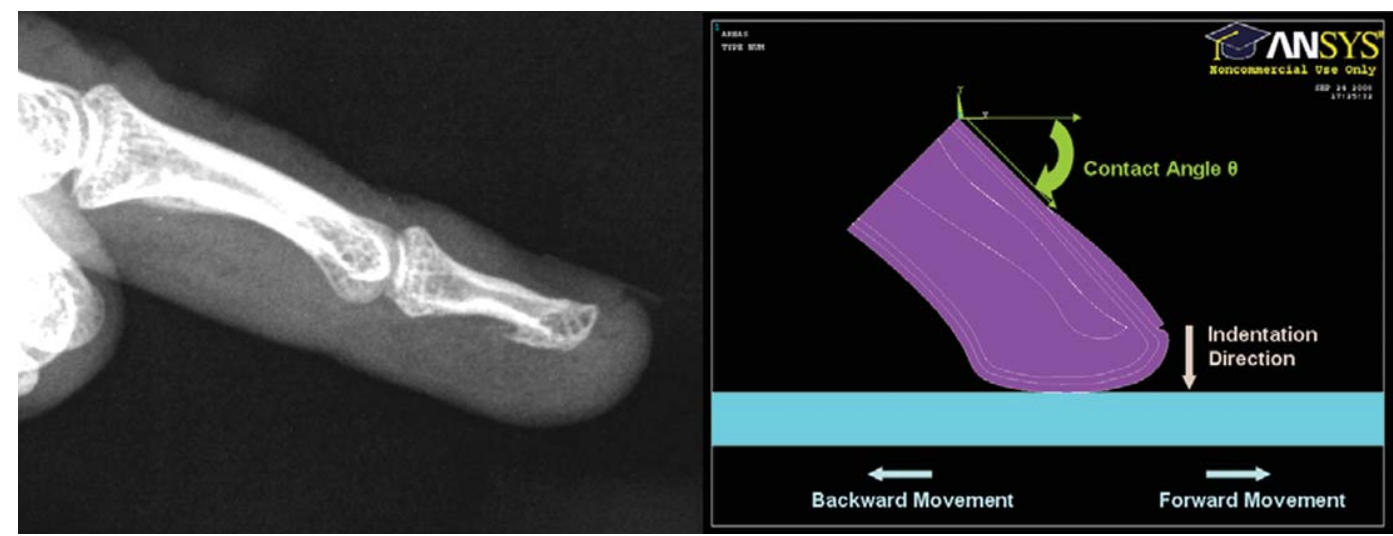

Fig. 6. (Left) Lateral view MR image (adapted from [17]) - (Right) Biomechanical inhomogeneous model of fingertip. 


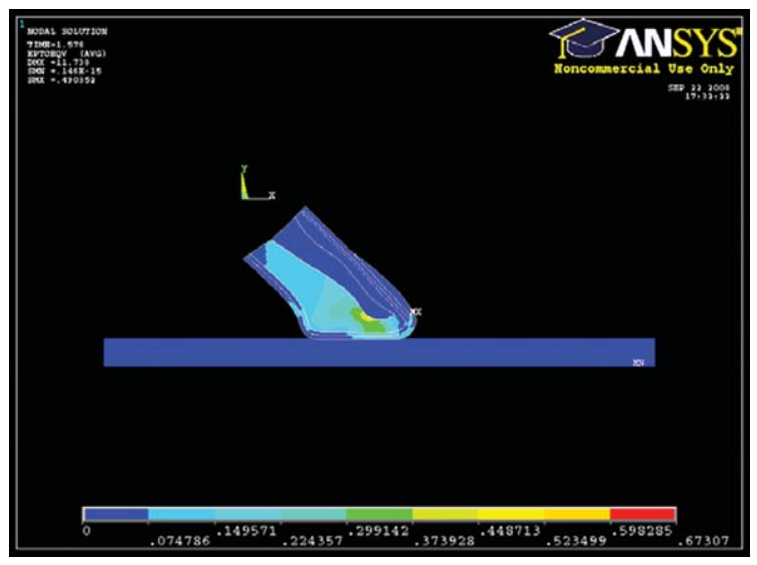

Fig. 7. The strain energy distribution during the simulation sampled at an arbitrary moment of proximal-to-distal movement of the fingertip on the surface.

Lagrange method was selected for this problem with a node to surface contact model. As explained in section 3 , the dynamic coefficient of friction was approximated to be equal to the static friction coefficient. In order to avoid divergence of numerical solutions, number of iterations and substeps was chosen by the software. For simulation, other required parameters, such as maximum contact friction, coefficient of friction and fingertip speed, were set according to Tables 1 and 2 for different surfaces.

The simulation procedure was performed first by a pre-indentation of $2 \mathrm{~mm}$ for the stickiness evaluation experiment and $1.5 \mathrm{~mm}$ for the slipperiness evaluation, before the dynamic touch phase. The neural response was approximated by strain energy density [14] computed at a typical SAII receptor location under the skin ( $\sim 2 \mathrm{~mm}$ under skin adapted from dimensions of the model of Maeno et al. [12] in dermis layer near the boundary with subcutaneous fat). Figure 7 portrays

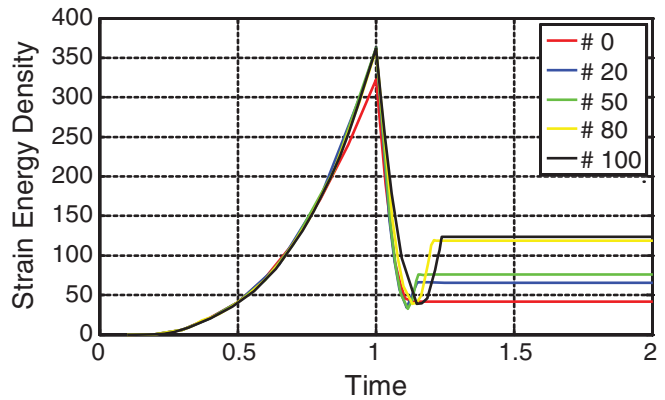

the strain energy distribution during the simulation sampled at an arbitrary moment of proximal-to-distal movement of the fingertip on the surface. The selected point lies approximately on the central axe of the contact area.

Figure 8 shows the predicted dynamic response of the SAII receptor for the simulated contact of the fingerpad with sample surfaces described in previous sections using measured data in Tables 1 and 2. The first second of simulation in all of the curves correspond to pre-indentation phase before reaching a steady-state response during the dynamic touch. Movement of fingertip was imposed on bone by assuming that in a free exploration task, it is the subject who controls the movement of the bone as a part of the skeleton system.

As explained in psychophysical experiment guidelines, the direction of fingertip movement for two categories were distinct (distal-to-proximal for the slipperiness evaluation and proximal-to-distal for the assessment of the stickiness). The effect of this distinction appears in the magnitude of local strain energy density as can be observed in the results for the two experiments even in the presence of a difference in preindentation depth as described above. In other words, with equal depth of indentation, order of strain energy density is much higher for backward movement of the fingertip than for forward movement. This is assumed to be due to the fingertip's biomechanical structure when coming in contact with a frictional surface, and the difference in contact tangential forces produced when keeping the indentation depth constant.

The steady-state part of the predicted neural response is the basis of further discussions in this article. Some geometrical micro-features of the fingerpad such as epidermal ridges or dermal papillae are not considered in this study, since their presentation seems not to have any significant contribution to

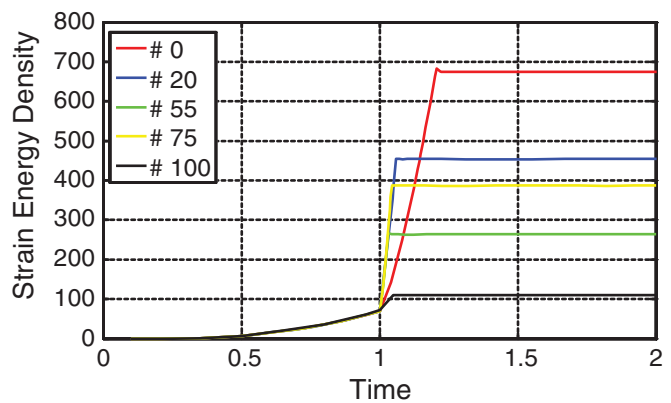

Fig. 8. Prediction of neural response of a typical SAII mechanoreceptor in controlled virtual experiments for evaluation of stickiness (left) and slipperiness (right). 

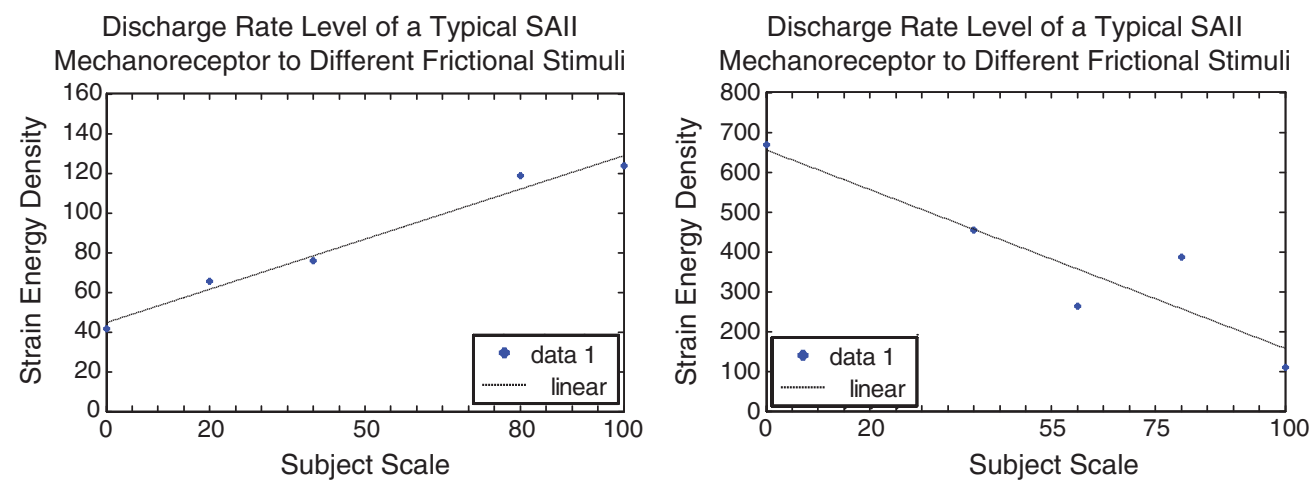

Fig. 9. Correlation between steady-state response of a SAII receptor with subject's scale of stickiness (left) and slipperiness (right).

predicted response. Rather, they tend to complicate the model and computational aspects such as the number of elements used because of their small dimensions. Moreover, previous literature does not appreciate the importance of these features in the mechanics of the sense of touch (e.g. [5]). On the other hand, the parameter of interest in this study is the surface friction and the behaviour of the surface is not considered to be "rough".

\section{Discussion}

Quantification of subjective evaluation of friction by the sense of touch is important for many applications in robotics, rehabilitation, virtual reality and development of friction based tactile displays. In order to achieve this goal, it is necessary to develop a biomechanical model of the fingerpad to investigate the response of individual mechanoreceptors. The fidelity of such a model can be verified if the predicted response by the model can be correlated with neurophysiological or psychophysical experimental data.

The aim of this study is to compare the anticipated reaction of a SAII receptor using the proposed 2D finite element model of fingerpad-surface contact with psychophysical subjective evaluation of the same stimuli. In order to abridge the complicated way of constructing the desired model, including estimation of precise geometrical features of fingerpad and identification of biomechanical structure and physical parameters of subcutaneous tissues, the idea is to employ physical dimensions and values from the literature.

The primary results of this approximate model, such as contact forces, can be checked with real data in order to avoid exaggeration. On the other hand, the raw mechanical results routinely need to be normalized to correlate with recorded neural response.

Another important assumption in this study is the linear relation between peripheral neural response of local mechanoreceptors and central nervous system judgement on frictional properties. Such an assumption was implicitly made by Smith et al. [13] through the investigated relation between contact forces and subjective judgements. Instantaneous variations of exerted normal forces by the user and fingertip speed in unconstrained exploration were not included in the simulations because of their insignificant effect in this study.

Contact parameters (coefficient of friction, $\mu$, and maximum contact friction, $\tau_{\max }$ ) required to perform simulations were computed from captured contact forces in the experimental setup for different sample surfaces used for tactile sense research. As explained previously, it is assumed that neural spikes density of individual mechanoreceptors correlate with mechanical strain energy density at the same locations. Hence, the relation between strain energy density at the depth of a typical SAII receptor, which is most sensitive to tangential force stimuli, and subjective evaluation of different frictional stimuli is sought. Constant values of steady-state responses of the selected mechanoreceptor in the exploratory simulations described in the previous section are depicted in Fig. 9 with corresponding subject scales of frictional properties of the surfaces. Subject scales are distinguished from index numbers of samples in the figure.

As a conclusion from the results, the linear relationship between the increase or the decrease of strain energy density at the location of the SAII mechanoreceptor and subject's scale of the related stimuli is kept. 
For the sticky property evaluation, the increase of local strain energy density is followed by the increase of the score given by the subject while in the slipperiness assessment; the subject has given a higher score to the stimulus making a lower level of local strain energy (more slippery stimulus) except for the 3rd sample (\#55). This anomaly is because of the lower captured coefficient of friction for this sample compared to the 4 th one (\#75) in most of the exploration course as can be seen in Fig. 4. However, it does not conflict with the conclusion since the subject decided on the given score after some exploratory trials, while the contact forces were captured once and this reduction in friction coefficient may be due to the instantaneous moisturizing of the subject's skin.

\section{Conclusion}

The nonhomogeneous biomechanical finite element model of fingertip proposed in this paper was employed to predict neural response of sensitive mechanoreceptors to frictional stimuli (Slowly Adapting SAII receptors under the glabrous skin). In order to feed the model with physical parameters intervening subjective assessment of friction, an experimental setup was proposed. In this setup, subjects were asked to quantify their judgement on surface stickiness or slipperiness of sample standard stimuli. Mechanical contact parameters such as friction coefficient or tangential contact forces were captured at the same time to feed the model. Neural firing rates of neural receptors were estimated during the dynamical simulation of fingertip touching a surface with the same measured physical parameters. These neural discharge rates were then compared to subjective scales of corresponding surfaces.

According to the simulation results, the biomechanical model predictions of the neural response and in consequence, subjective assessment of frictional properties of surfaces were consistent with the results of psychophysical experiments. In other words, strain energy was higher at the level of mechanoreceptors for more sticky samples and was lower for more slippery samples.

\section{Acknowledgement}

This work has been carried out within the framework of the INRIA Alcove project however the findings and conclusions in this report are those of the author and do not necessarily represent the views of the corresponding institute. Also, the author would especially like to thank Dr. Nigel Stepp for taking the time to review this manuscript.

\section{References}

[1] M. Abdolvahab, Rendering edge enhancement tactile phenomenon by friction variation in dynamic touch, Journal of Biomechanics 44(1) (2011), 92-96.

[2] S. Crochemore, A new reference frame for tactile perceptions: Sensotact. The 5th Rose Mary Pangborn Meeting, Boston, MA, USA, 2003.

[3] K. Dandekar, B.I. Raju and M.A. Srinivasan, 3-D finiteelement models of human and monkey fingertips to investigate the mechanics of tactile sense, J Biomech Eng 125 (2003), 682-691

[4] B.B. Edin, Quantitative analysis of static strain sensitivity in human mechanoreceptors from hairy skin, J Neurophysiol 67 (1992), 1105-1113.

[5] G.J. Gerling and G.W. Thomas, Fingerprint lines do not affect SA-I mechanoreceptor response, Somatosensory and Motor Research 25(1) (2008), 61-76.

[6] T. Harrington and M.M. Merzenich, Neural coding in the sense of touch: Human sensations of skin indentation compared with the responses of slowly adapting mechanoreceptive afferents innervating the hairy skin of monkeys, Exp Brain Res 10(3) (1970), 251-264.

[7] S. Johansson and G. Westling, Roles of glabrous skin receptors and sensorimotor memory in automatic control of precision grip when lifting rougher or more slippery objects, Exp Brain Res 56 (1984), 550.

[8] S. Johansson and G. Westling, Signals in tactile afferents from the fingers eliciting adaptive motor responses during the precision grip, Exp Brain Res 66 (1988), 141-154.

[9] K.O. Johnson, The roles and functions of cutaneous mechanoreceptors, Current Opinion in Neurobiology 4 (2001), 455-461.

[10] K.O. Johnson, T. Yoshioka and F. Vega-Bermudez, Tactile functions of mechanoreceptive afferents innervating the hand, J Clin Neurophysiol 17 (2000), 539-558.

[11] M. Knibestol and A.B. Vallbo, Single unit analysis of mechanoreceptor activity from the human glabrous skin, Acta Physiol Scand 80 (1970), 178-195.

[12] T. Maeno, K. Kobayashi and N. Yamazaki, Relationship between the structure of human finger tissue and the location of tactile receptors, JSME Int J 41 (1998), 94-100.

[13] A.M. Smith and S.H. Scott, Subjective scaling of smooth surface friction, J Neurophysiol 75(1) (1996), 1957-1962.

[14] M.A. Srinivasan and K. Dandekar, An investigation of the mechanics of tactile sense using two-dimensional models of the primate fingertip, J Biomech Eng 118 (1996), 48-55.

[15] M.A. Srinivasan, J.M. Whitehouse and R.H. LaMotte, Tactile detection of slip: Surface microgeometry and peripheral neural codes, J Neurophysiol 63 (1990), 1323-1332.

[16] M. Tada, N. Nagai, H. Yoshida and T. Maeno, Iterative FE analysis for non-invasive material modeling of a fingertip with layered structure, Proceedings of Eurohaptics 2006, 2006. 
[17] Van Ruyssevelt, E.A. Christian, Patrick and Vranckx, Subungual Glomus Tumor: Emphasis on MR angiography, Am J Roentgenol 182(1) (2004), 263-264.

[18] Q. Wang and V. Hayward, In vivo biomechanics of the fingerpad skin under local tangential traction, Journal of Biomechanics 40(4) (2007), 851-860.

[19] G. Westling and R.S. Johansson, Responses in glabrous skin mechanoreceptors during precision grip in humans, Exp Brain Res 66 (1987), 128-140.
[20] W.D. Willis and R.E. Coggeshall, Sensory mechanisms of the spinal cord: Volume 1: Primary afferent neurons and the spinal dorsal horn. Springer (2004), 70-72.

[21] J.Z. Wu, K. Krajnak, D.E. Welcome and R.G. Dong, Analysis of the dynamic strains in a fingertip exposed to vibrations: Correlation to the mechanical stimuli on mechanoreceptors, Journal of Biomechanics 39 (2006), 2445-2456. 

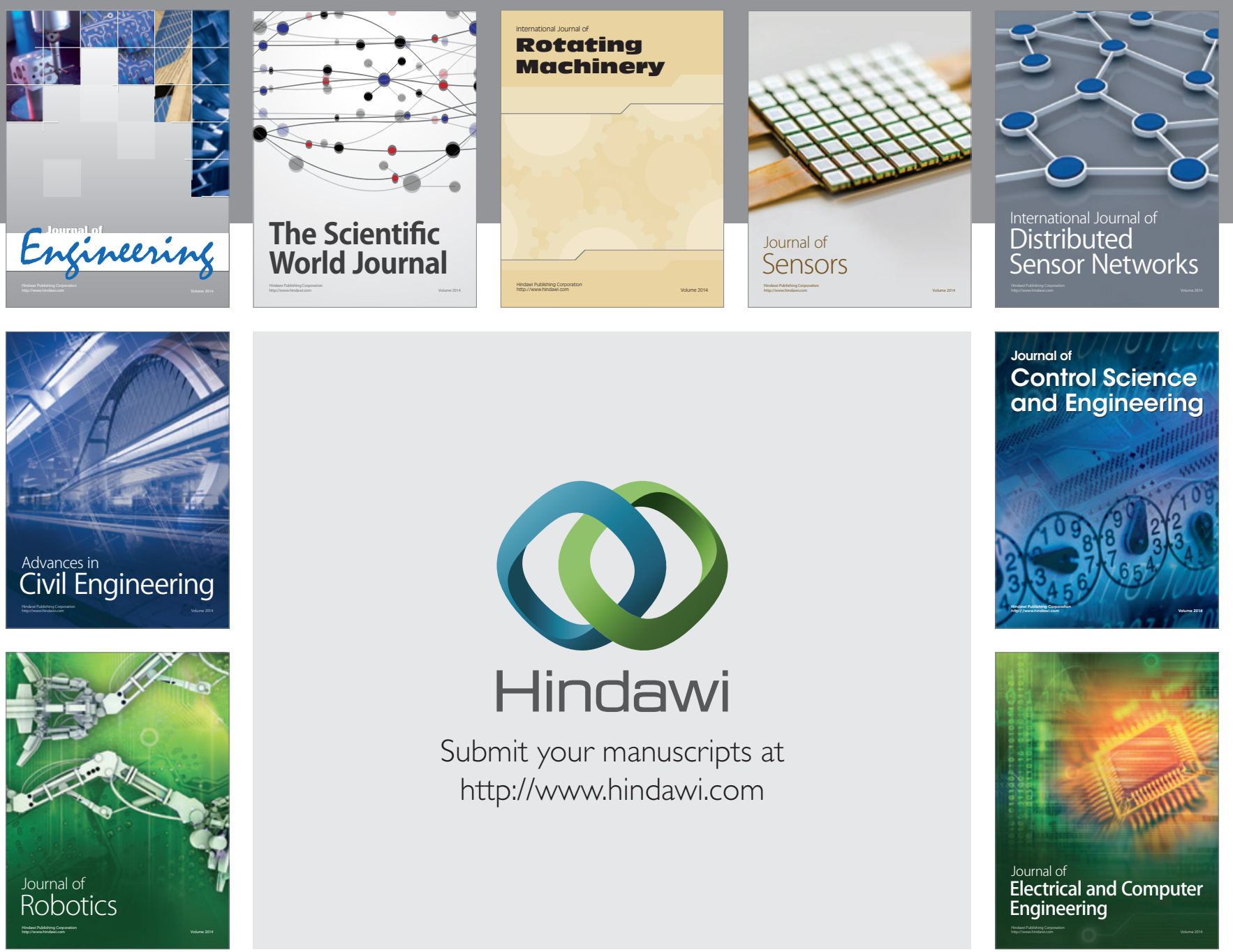

Submit your manuscripts at

http://www.hindawi.com
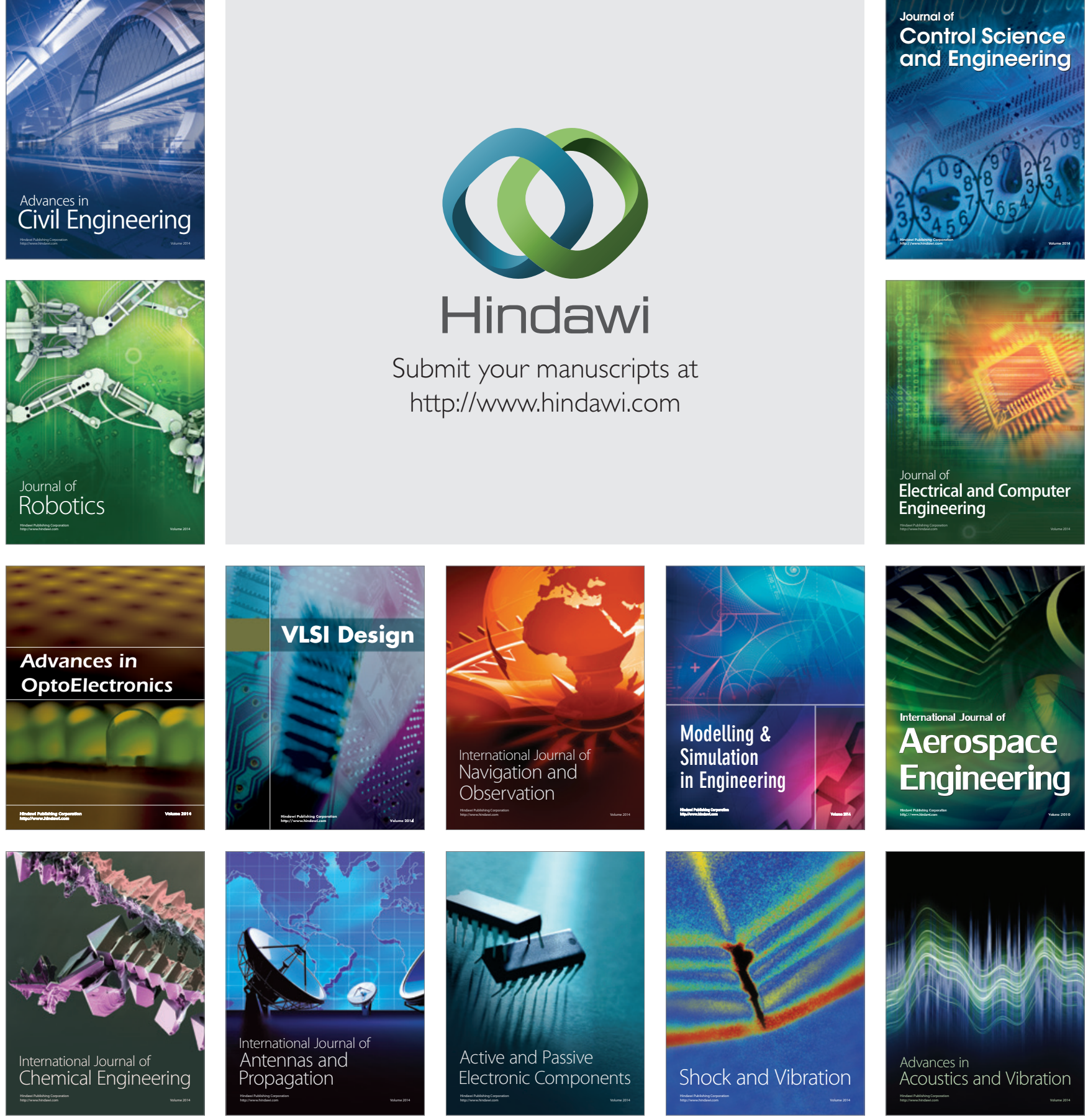